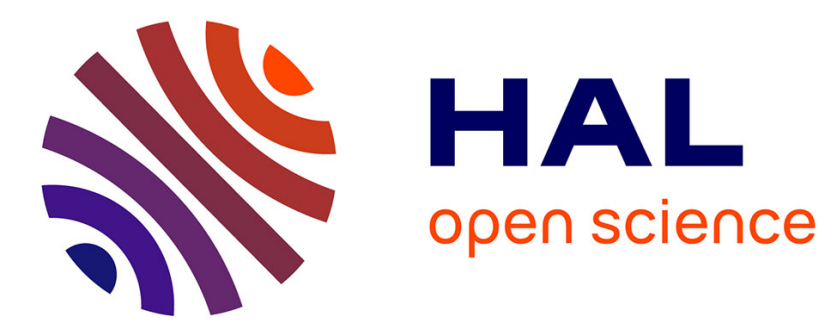

\title{
Tomographie ultrasonore par traitement d'images radiales: application à l'imagerie médicale
}

\author{
B. Migeon, Pierre Vieyres, P. Marche
}

\section{To cite this version:}

B. Migeon, Pierre Vieyres, P. Marche. Tomographie ultrasonore par traitement d'images radiales: application à l'imagerie médicale. Journal de Physique IV Proceedings, 1994, 04 (C5), pp.C5-1297C5-1300. 10.1051/jp4:19945288 . jpa-00253000

\section{HAL Id: jpa-00253000 https://hal.science/jpa-00253000}

Submitted on 1 Jan 1994

HAL is a multi-disciplinary open access archive for the deposit and dissemination of scientific research documents, whether they are published or not. The documents may come from teaching and research institutions in France or abroad, or from public or private research centers.
L'archive ouverte pluridisciplinaire HAL, est destinée au dépôt et à la diffusion de documents scientifiques de niveau recherche, publiés ou non, émanant des établissements d'enseignement et de recherche français ou étrangers, des laboratoires publics ou privés. 


\title{
Tomographie ultrasonore par traitement d'images radiales : application à l'imagerie médicale
}

\author{
B. MIGEON, P. VIEYRES et P. MARCHE
}

Laboratoire Vision et Robotique, 63 Avenue de Lattre de Tassigny, 18020 Bourges cedex, France

\begin{abstract}
This work presents a new technique of image reconstruction by backscattering ultrasound tomography using radial images. It is based on the fact that a B mode echographic image, although incomplete or noisy, always contains more information than an image obtained by a projection technique. The new technique is to reconstruct a slice from radial echographic images of this same slice using classic echographic probes. The new method of "Moyennes Maximisées" (maximized-averages) combines the advantages of the method of "Maxima" and the method of "Moyenne" (average). This method was tested and applied in-vitro for the reconstruction of a slice of a fetus thigh. It offers a better image quality that increases with the number of radial images $N$, and enables to distinguish between various anatomical parts of the slice.

Key Words : Backscattering Ultrasound Tomography, "Maximized Averages", Ultrasound Medical Imaging
\end{abstract}

\section{INTRODUCTION.}

La technique ultrasonore est maintenant très couramment utilisée en imagerie médicale pour l'exploration des corps mous (gynéco-obstétrique, foie, rein, coeur) grâce à l'échographie standard (mode-B) [1]. Toutefois, dès que l'application demande une qualité d'image accrue, comme c'est le cas pour l'investigation du sein par exemple, ou bien l'exploration d'une coupe contenant des structures très échogènes, comme c'est le cas pour l'investigation des os longs des membres, l'échographie classique est insuffisante et fait place à la reconstruction tomographique ultrasonore. La recherche dans ce domaine existe depuis une vingtaine d'années et un très grand nombre de techniques basées sur l'utilisation de projections a vu le jour, que ce soit en mode transmission $[2,3]$ ou en mode réflexion $[4,5]$.

Ce travail présente le principe de la reconstruction tomographique ultrasonore en mode réflexion à partir d'images radiales, qui utilise des images obtenues par échographie classique, et non plus à partir de projections. La méthode des moyennes maximisées décrite ici, combine les avantages de deux méhodes plus simples, et permet d'obtenir une bonne qualité d'image. Cette méthode a été testée par expérimentation in vitro sur une pièce anatomique de nouveau-né au niveau de la cuisse. 


\section{PRINCIPE DE LA RECONSTRUCTION ET METHODES EXISTANTES.}

\section{1 Principe}

La reconstruction tomographique ultrasonore à partir d'images radiales repose sur l'idée de base qu'une image échographique, même bruitée et donnant une représentation incomplète du plan de coupe exploré, est forcément plus riche d'information qu'une projection. Dès lors, le principe consiste à reconstruire le plan de coupe investi à partir d'images radiales et non plus à partir de projections.

Soient $\mathrm{I}(\mathrm{x}, \mathrm{y})$ l'image à reconstruire, et $\mathrm{L}(\mathrm{x}, \mathrm{y})$ la luminance en niveau de gris $\mathrm{du}$ pixel de coordonnées $(x, y)$ sur $I$. Les méthodes de tomographie ultrasonore à partir d'images radiales ont pour but de reconstruire l'image $I(x, y)$ à partir des $N$ images radiales prises selon $\mathrm{N}$ directions radiales d'investigation angulairement équidistantes de $\alpha=2 \pi / N$. Elles consistent à déterminer la luminance $L(x, y)$ en fonction des $N$ luminances $\operatorname{Li}(x, y)(i=1 . . N)$ des $N$ images radiales préalablement recallées entre elles. L'objet du recalage est de tourner chacune des images de l'angle adéquat en fonction de sa direction radiale d'investigation. Ainsi, le pixel de coordonnées $(x, y)$ de chacune des $N$ images correspond au même point réel de la coupe explorée.

\section{2 Méthode des maxima}

Cette méthode consiste à déterminer $\mathrm{L}(\mathrm{x}, \mathrm{y})$ en sélectionnant la valeur maximale parmi les $\mathrm{N}$ luminances $\mathrm{Li}(\mathrm{x}, \mathrm{y})$ des $\mathrm{N}$ images radiales [6].

$$
\forall(x, y) \in I \quad L(x, y)=\operatorname{Max}_{1} I_{1}(x, y) \quad i=1 \ldots N
$$

Avec un nombre d'images radiales faible, elle permet de représenter les structures hyperéchogènes présentes sur au moins une image radiale, telles que les structures osseuses. En revanche, elle est extrèmement sensible au bruit et, lorsque $\mathrm{N}$ augmente, l'interprétation diagnostique des autres structures devient très difficile [7].

\section{3 Méthode de la moyenne}

Cette méthode consiste à calculer $\mathrm{L}(\mathrm{x}, \mathrm{y})$ en faisant la moyenne des $\mathrm{N}$ luminances $\mathrm{Li}(\mathrm{x}, \mathrm{y})$ des images radiales

$$
\forall(x, y) \in I \quad L(x, y)=\frac{1}{N} \sum_{i=1}^{N} L_{i}(x, y) \quad i=1 \ldots N
$$

Elle permet une attténuation du bruit, d'autant meilleure que $\mathrm{N}$ augmente, comme on peut s'y attendre s'agissant d'une moyenne. Mais en contrepartie, elle atténue sensiblement toutes les structures (surtout hypoéchogènes et moyennement échogènes)[7]. Ainsi, lorsque $\mathrm{N}$ augmente, les structures hyperéchogènes se dégagent et les autres structures sont difficilement discernables car trop atténuées ou noyées dans un flou ambiant.

\section{METHODE DES MOYENNES MAXIMISEES.}

La méthode des moyennes maximisées combine la méthode des maxima et la méthode de la moyenne, afin de tirer parti de leurs avantages respectifs. Elle consiste à appliquer la méthode des maxima sur $\mathrm{N}$ nouvelles images radiales intermédiaires 
construites de façon sélective par la méthode de la moyenne. Chaque image radiale intermédiaire est reconstruite par la méthode de la moyenne appliquée à $(2 m+1)$ images radiales d'origine, à savoir l'image correspondant à la même direction radiale d'investigation, et $m$ images radiales situées de part et d'autre. Ces $(2 m+1)$ images sont choisies de telle sorte que les "points de vue" pour l'exploration selon ces directions radiales, appartiennent de façon exclusive au demi-plan délimité par une droite perpendiculaire à la direction radiale considérée [7].

La formulation de cette méthode est la suivante:

$$
\begin{gathered}
\forall(x, y) \in I \quad L(x, y)=\max _{i} L_{i}^{\prime}(x, y) \quad i=1 . . N, N \geq 5 \\
\text { avec } \quad L_{i}^{\prime}(x, y)=\frac{1}{2 m+1} \sum_{j=n_{i}}^{n_{i+1}} L_{k(j)}(x, y) \quad i=1 . . N \\
\left\{\begin{array}{ll}
n_{i}=i-m \\
n_{i+1}=i+m
\end{array} \quad m=\left\{\begin{array}{lll}
E\left(\frac{N}{4}\right) & \text { si } E\left(\frac{N}{4}\right) \neq \frac{N}{4} \\
\frac{N}{4}-1 & \text { sinon }
\end{array} \quad k(j)=\left\{\begin{array}{lll}
j & \text { si } & j \in[1 . N] \\
j-N & \text { si } & j>N \\
j+N & \text { si } & j<1
\end{array}\right.\right.\right. \\
\text { où } E(z) \text { est la partie entière de } z
\end{gathered}
$$

Dans une direction radiale, la reconstruction sélective par la méthode de la moyenne permet d'obtenir une image intermédiaire débruitée et représentant correctement tous les types de structures visibles selon cette direction radiale. La méthode des maxima appliquée aux $\mathrm{N}$ images intermédiaires permet de garder une bonne représentation des structures sur l'ensemble de la coupe. La figure 1 représente la reconstruction d'une coupe de cuisse de nouveau-né obtenue par expérimentation in-vitro, en utilisant la méthode des moyennes maximisées $(N=16)$. Cette méthode offre une bonne dynamique des niveaux de gris, et des contrastes bien établis entre les différentes structures moyennement échogènes et hyper-échogènes de la coupe étudiée. Les différentes structures présentes (fémur, masses musculaires, etc..) sont schématisées sur la figure 2.

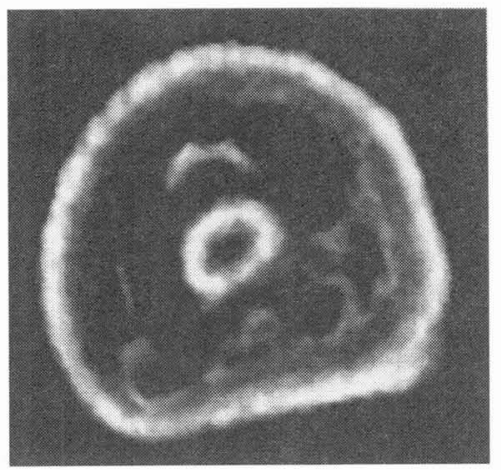

Fig. 1 : Reconstruction d'une coupe au niveau de la cuisse par la méthode des moyennes maximisées avec $\mathrm{N}=16$

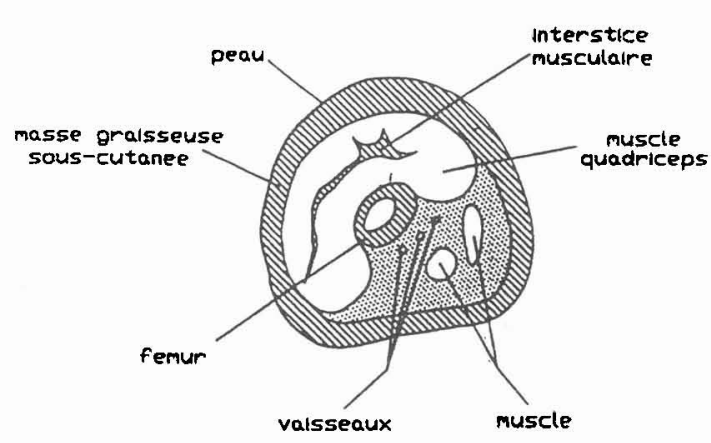

Fig. 2 : Schéma d'interprétation de la coupe présentée sur la figure 1 


\section{CONCLUSION.}

Nous avons présenté la méthode des moyennes maximisées qui s'inscrit dans le cadre de la reconstruction tomographique ultrasonore utilisant des images radiales. Elle combine les méthodes des maxima et de la moyenne pour profiter de leurs avantages respectifs. Elle offre une bonne qualité d'image, et permet de mettre en évidence les différentes structures anatomiques présentes au niveau d'une coupe de cuisse de nouveau-né.

\section{REFERENCES}

[1] Pourcelot L., Dynamique cardio-vasculaire foetale et néonatale:EchographieDoppler (Editions Masson, 1991)

[2] Greenleaf J. F., Bahn R. C., "Clinical imaging with transmissive ultrasonic computerized tomography". IEEE Trans. Biomed. Eng., vol. BE 28 (1980), 177-186.

[3] Greenleaf J. F., Grisvold J. J., Bahn R. C., "Computered transmission ultrasound tomography". Med. Prog. Technol., vol 9 (1982), 165-170.

[4] Hiller D., Ermert H., "System analysis of ultrasound reflection mode computerized tomography". IEEE Trans. Sonics Ultrason., vol. SU 31 (1984), 240250.

[5] Dines K. A., Goss S. A., "Computed ultrasonic reflection tomography". IEEE Trans. Ultrason. Ferroelectr. Freq. Contr., vol UFFC 34, n 3 (1987), 309-318.

[6] Migeon B., Marche P., "Reconstruction de plans de coupe en échographie par traitement d'images radiales". ITBM, vol 13, n³ (1992), 292-304.

[7] Migeon B., "Reconstruction 2D et 3D d'os longs des membres par traitement d'images échographiques". Thèse de Doctorat. Université d'Orléans (1993). 